\title{
INFRARED DIAGNOSTICS FOR MEASURING FLUID AND SOLID MOTION INSIDE MEMS
}

\author{
Gengxin Han, James C. Bird, K. Johan A. Westin \& Kenneth S. Breuer \\ Division of Engineering, Box D Brown University \\ Providence, RI 02912. USA
}

\begin{abstract}
A new velocimetry system has been developed for use in microdevices that incorporate silicon as their structural material. The system is designed to illuminate and measure particle and surface motions using infrared wavelengths, taking advantage of the fact that silicon is largely transmissive to light with wavelength above 1 micron. The system allows us to observe motion inside silicon-based microdevices, which are otherwise opaque to light at visible wavelengths. By analyzing these images using both timeof-flight and phase-locked techniques, we can make quantitative measurements about the position and speed of internal surfaces and the motion of fluids inside complex microfabricated devices.
\end{abstract}

\section{INTRODUCTION}

The rapid growth and significant progress in the development of microfluidic devices has revealed a need for measurement techniques to measure the flow inside microdevices with micronscale resolution. Non-invasive diagnostics, such as Particle Image Velocimetry (PIV) [1] are well-suited to this task and in recent years, micro-PIV techniques have been described $[\mathbf{6 , 8}]$ which press conventional methods to the limits of optical resolution. PIV is one example of a time-of-flight measurement, in which the velocity of an object is inferred by measuring its displacement during a known time period. The displacement is computed from two images, each exposed with a short laser pulse and taken in rapid succession. If the objects of interest are small particles suspended in a fluid, the velocity field can be measured. However the technique is not restricted to suspended particles and can also be used on any feature that can be tracked in a pair of images. A second mode of measuring motion is by stroboscopic illumination of an object in harmonic motion. In general, this technique cannot be used for fluid motion, because, even if the flow is harmonic, suspended particles are unlikely to follow closed paths. However, this phase-locked technique has been used to great success in the measurement of the motion of harmonically-forced MEMS devices such as accelerometers, etc. [4].

As the complexity of the microdevices increases, the ability to provide optical access through a transparent surface becomes increasingly difficulty to ensure. The use of transparent materials (glass, Pyrex, plastic, etc.) often represents a compromise to the fabrication sequence, as these materials are not as structurally or thermally robust as single-crystal silicon or silicon carbide and cannot be processed with as much flexibility as is available for silicon-based substrates. In addition, the fabrication methods for these materials tend to be less precise, compromising precision fabrication. Lastly, as multi-layer microfabricated silicon structures become increasingly common, the requirement to incorporate a glass window for visualization purposes is often impossible to accommodate without further complication to the fabrication sequences - a complication that is usually unacceptable.

Although visible light is no longer suitable as an illumination source for a micro-PIV system, the absorption coefficient of silicon falls sharply in the near infrared regime, and is effectively transparent at wavelengths between 1.1 and 2.5 microns. This property implies that infrared (IR) light might be a desirable illuminating source for PIV system if one would like to visualize motion inside a silicon-based microdevice. The present paper explores the idea of using IR illumination to measure motion inside silicon devices.

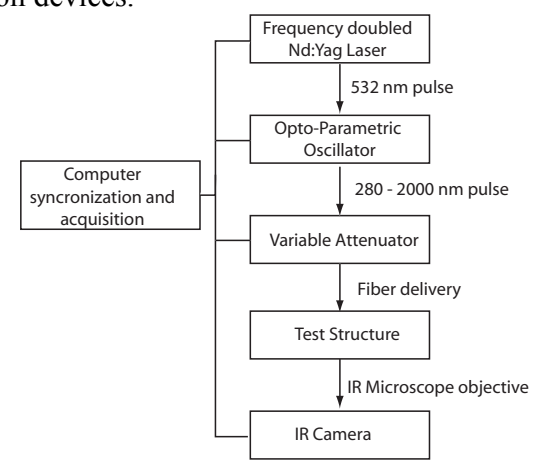

Figure 1. Overall schematic of IR micro motion system.

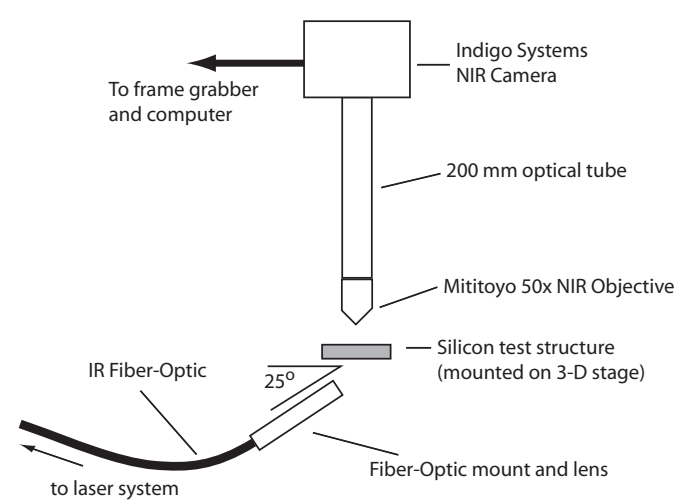

Figure 2. Schematic of the optical configuration for micro-motion measurement.

\section{BACKGROUND THEORY}

The general optical system is outlined in Figure 1 and Figure 2. The device is illuminated using a twin Nd:YAG laser system which emits a pair of $4.5 \mathrm{~ns}$ pulses at $1064 \mathrm{~nm}$. Although we could use this wavelength, the transmissivity of silicon is not optimal here, and higher wavelengths are more desirable. To achieve this, the beam is frequency-doubled to $532 \mathrm{~nm}$ and then directed through an Opto-Parametric-Oscillator (OPO) - a nonlinear crystal system that enables the laser pulse to be tuned to a wavelength anywhere between $300 \mathrm{~nm}$ and $2000 \mathrm{~nm}$. In the present case, we typically tune the laser to $1200 \mathrm{~nm}$, where silicon reaches a high level of transparency. The pulse is then coupled to a fiber-optic delivery system and directed to the target, which is floodilluminated by the two laser pulses. The angle at which the light hits the target is quite important, and depends on what is being 
illuminated. For solid surface measurement, we have found that illumination in pure transmission mode is the most effective, in which case the laser is positioned directly below the sample, at ninety degrees to the focal plane. For small particle illumination typical of PIV, a shallow angle of approximately 25 degrees with respect to the focal plane was found to be optimal, although the precise dependence of this is still being quantified more closely.

The scattered light is collected by a Near Infrared (NIR) microscope objective mounted on a $200 \mathrm{~mm}$ corrected tube and imaged using an Indigo Systems Indium Gallium Arsenide (InGaAs) NIR camera. This camera has a 320x256 pixel array (30 micron pixel size) with excellent sensitivity between 900 and 1700 $\mathrm{nm}$. Different microscope objectives have been used for different studies. For PIV measurements, a 50x 0.42NA Mititoyo objective has been used. This optical arrangement gives a field of view of 192 by 154 microns, which although small, gives good resolution of the small particles that are imaged in PIV measurements. For the solid surface motion study described in this paper, a 20x 0.3 NA objective was used to achieve a larger field of view (at the cost of spatial resolution).

The resultant images are transferred at $60 \mathrm{~Hz}$ to a data acquisition computer. Although the NIR camera is a video-rate device and cannot be triggered, as can most modern CCD systems, the laser pulses are synchronized to the video sync signal and can be adjusted to fall anywhere within the video frame. In the case of phase-locked operation, the position of the laser pulse in the frame is not important, as long as it doesn't fall on the inter-frame boundary, in which case a dead image will result. However, in the case of time-of-flight measurements, the laser timing is adjusted so that the two laser pulses straddle adjacent video frames. For the Indigo camera, this inter-frame boundary is $0.12 \mathrm{~ms}$ in duration, so that the separation between the two laser flashes can be no shorter than $0.12 \mathrm{~ms}$-more than adequate for most low-speed motions. Higher speed activity can be captured either with dual-illumination of a single frame or by using two cameras externally synchronized to be out of phase. Triggered IR cameras or interline cameras which can store two images in quick succession may be available in the near future to ease this concern.

\section{FLUID VELOCITY MEASUREMENTS}

A significant difference between visible micro-PIV and the IR micro-PIV system is the particle scattering technique used. In a visible PIV system, one can use an inelastic technique, such as epifluorescence, to image submicron particles. This has the advantage that background reflections at the excitation wavelength can be filtered out by the use of a dichroic filter, greatly enhancing the sensitivity of the image-gathering optics and enables the use of small particles, which can track the flow very accurately. In contrast, in the IR system, fluorescent particles with both absorption and emission bands in the IR regime do not exist (to our knowledge), and this mandates the use of elastic scattering techniques to image the particles. Laser scattering from small particles is highly dependent on the particle size and wavelength, and the scattered intensity $(I)$ varies like [2]:

$$
I \propto d^{6} / \lambda^{4}
$$

where $d$ is the particle diameter and $\lambda$ is the wavelength of the recording light. From which we see, there is a tradeoff between higher wavelengths to achieve greater silicon transmissivity, and greater sensitivity in the NIR camera, (which also increases at higher wavelengths), and using lower wavelengths to maximize the scattering intensity and to improve the point spread function size. A compromise wavelength of $1200 \mathrm{~nm}$ was found to work well, although some results in this paper were also obtained at $1064 \mathrm{~nm}$ (before the OPO was fully operational). The intensity penalty due to wavelength can also be offset by using particles of larger diameter, $d$.

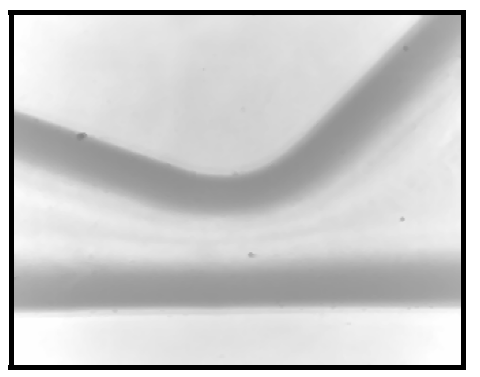

Figure 3. IR image of micronozzle throat using broad band illumination without flow. The shadows are due to the diffraction in the silicon-air transition.

The geometry tested was a converging-diverging flow inside a silicon micronozzle, shown in Figure 3. The device was fabricated by etching the nozzle geometry 300 microns deep into a 500 micron-thick silicon wafer. A second silicon wafer was fusionbonded to the first in order to cap the structure. The device is thus opaque to visible light. The nozzle shown in Figure 3 has a throat width of $40 \mu \mathrm{m}$. The measurements reported here were taken downstream of the throat where the flow expands to a channel $300 \mu \mathrm{m}$ deep by $1000 \mu \mathrm{m}$ wide.

The water flow was seeded $(0.06 \%$ by volume $)$ with 1 micron uncoated polystyrene particles with a refractive index of 1.56 at $589 \mathrm{~nm}$. The relative refractive index is 1.17 (compared to water). Smaller particles would be preferable, but the intensity increases strongly with particle diameter (see above), and this was found to give good particle visibility.

In a micro-PIV system, the spatial resolution of the system is given by the diameter of the diffraction-limited point spread function, $d s$, in the image plane, which is given by:

$$
d_{s}=2.44 M \frac{\lambda}{2 N A}
$$

where $M$ is the total magnification of the microscope, and $N A$ is the numerical aperture of the objective lens $[\mathbf{2 , 6}$. At a wavelength of $560 \mathrm{~nm}$, with a 1.4 NA $60 \mathrm{x}$ objective, Meinhart et al. report $d_{s}=$ $29.3 \mu \mathrm{m}[\mathbf{8}]$. For the same lens system (which will operate in the Near IR, as long as it is not anti-IR coated), but using light at 1.2 microns, this is degraded to 62 microns. For the $50 \mathrm{x}, 0.42 \mathrm{NA}$ objective used in the current study, the point-spread diameter is 174 microns. This is considerably larger than the optimal lens system and is primarily due to the low NA of the current lens. However, the low NA has a significant advantage - it has a large working distance, which is important for imaging inside thick silicon devices, and convenient for this preliminary study.

If we track a 1-micron particle, then, following Adrian [1] and Meinhart et al. [6], the effective diameter, $d_{e}$, projected on the image plane can be expressed as

$$
d_{e}=\left(M^{2} d_{p}^{2}+d_{s}^{2}\right)^{1 / 2},
$$


which yields $d_{e}=181$ microns, compared with approximately 30 microns reported by Meinhart et al. for visible light micro-PIV. The spot size is dominated by the diffraction point spread. However, since the NIR camera has large pixel sizes (30 microns), this is not as bad as it seems, and the imaged particle is only six pixels wide - close to ideal for PIV sub-pixel interpolation. Assuming that we can achieve $1 / 10^{\text {th }}$ pixel accuracy in the PIV algorithms, this gives a spatial resolution of $360 \mathrm{~nm}$.

In the current experiment, by using two pulse separation times, $(\delta \mathrm{t}=0.125 \mathrm{~ms}$ and $17.5 \mathrm{~ms})$ we measured the velocity in two flow conditions: a) a high flow rate regime where the average nozzle throat velocity is $62 \mathrm{~mm} / \mathrm{s}$ and b) a low flow rate regime where the average nozzle throat velocity is $250 \mu \mathrm{m} / \mathrm{s}$. The interrogation areas chosen were a region close to the wall (for the high speed flow condition) and a region away from walls in the nozzle expansion (for the low speed flow condition). These are simply representative regimes, and no attempt was made to map the entire flow field at this time.

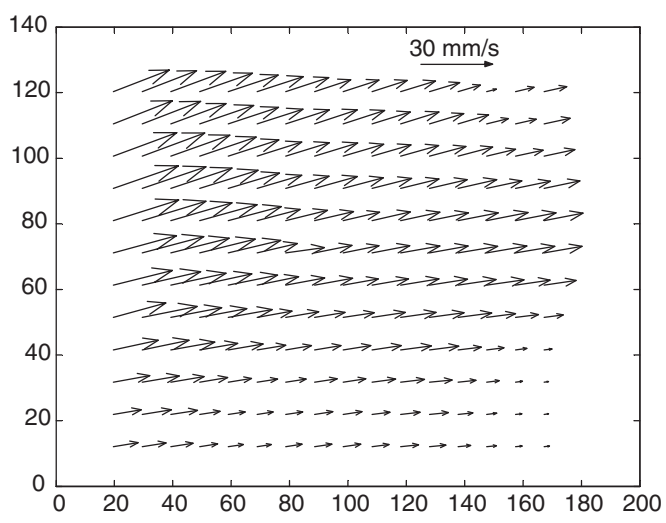

Figure 4. Velocity vectors for a high flow rate case. The lower part of the test area is close to the nozzle wall. The axis coordinates are in microns.

The image pair was analyzed using in-house PIV processing techniques, and selected results were confirmed using well-tested custom microPIV software [10]. The in-house PIV data processing techniques are based on the MATLAB-based image analysis software developed by Sveen [9] modified to include the crosscorrelation signal-to-noise ratio as a criterion during the ensemble averaging of velocity vectors. In processing the average, we first set an acceptable value of signal-to-noise ratio (SNR) and only pick up the vector whose correlation satisfies the SNR threshold participating in the average. The typical number of measurements included in an average is about 40 and only $7 \%$ of the total vectors were rejected due to poor SNR. The vector fields for the high speed condition are shown in Figure 4, computed using an interrogation window size of $64 \times 64$ pixels, and by overlapping the interrogation windows by $75 \%$. The lower axis represents the location of the solid wall where the velocity is zero. The focal place is in the middle of the nozzle, away from either the upper or lower nozzle boundaries. The dynamic range of the system (the ratio of the highest resolved speed to the lowest resolved speed) is limited by the pixel resolution of the images.

\section{SOLID SURFACE MOTION MEASUREMENT}

The IR micromotion system can also be used for detection of the position and velocity of a solid feature inside the silicon structure. If the motion of the solid object is harmonic, we can operate in "phase-locked" mode, in which the laser is locked to the frequency of the device (or some precisely control detuned frequency). This has been amply demonstrated using visible light by Freeman [4], by our own group, using IR illumination [5]. If, however, the motion of the solid object is not harmonic, there is no phase reference and a time-of-flight measurement technique, similar to the PIV technique is necessary. This is demonstrated using the rotation of a microturbine, fabricated as part of the MIT Microengine program [3]. The rotor consists of a $4 \mathrm{~mm}$ turbine disk, which can rotate in a stationary housing (supported by an air bearing). The completed microturbine is a complex device and is comprised of five silicon wafers bonded in a stack with the rotor forming the middle layer. The entire device is encased by silicon and is not accessible to visible light, save for a small access hole, for a fiber-optic speed sensor. One motivation for imaging the rotation is to determine both the speed of rotation and (more importantly) to image the details of any sub-synchronous motion ("whirl"), which can lead to rotor instabilities and ultimately to failure.

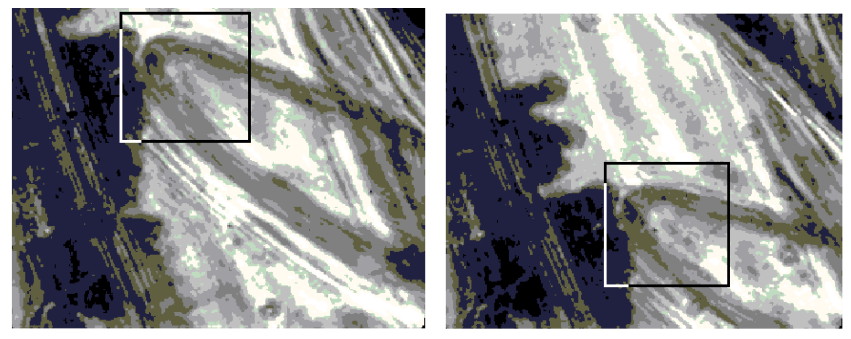

Figure 5. Pair of IR images taken using laser illumination, of a buried microrotor as it spins inside its rotor bearing. The size of each image is 480 by 384 (microns). The square boxes in each frame represent the initial interrogation areas used to compute translation and rotation.

Figure 5 shows a pair of images of the moving rotor taken using the laser illumination while it is spinning at low speed (approximately 5670 RPM). In the time-of-flight (or PIV) mode, the lasers are fired in rapid succession, and in this case the two flashes are separated by 150 microseconds. The motion of the rotor is clearly visible. In order to analyze this image pair both the translation and rotation of image $\mathrm{B}$ with respect to image A must be computed. The pictures are first cleaned up by removing dead pixels and overexposed pixels either by thresholding the image (reassigning pixels that have values that are below or above a given value) or by using a Median filter or an adaptive Wiener filter, both designed to remove high frequencies and "salt and pepper" noise. The images are also equalized so that their intensity distributions are matched.

The velocity of a feature in the image is determined as follows: A first-guess interrogation area (IA) is first selected on each image. This area should contain well-defined features that will be easy to track. Edges and other high-contrast features are most desirable. In addition, features that are highly non-isotropic (i.e. have clear orientation) are preferable in order to compute image rotation. In the example shown above, the leading edge of the rotor blade is selected as the interrogation area. The images are next linearly de-trended, windowed (using a Hamming window) and cross-correlated using an FFT-based cross-correlation routine. 


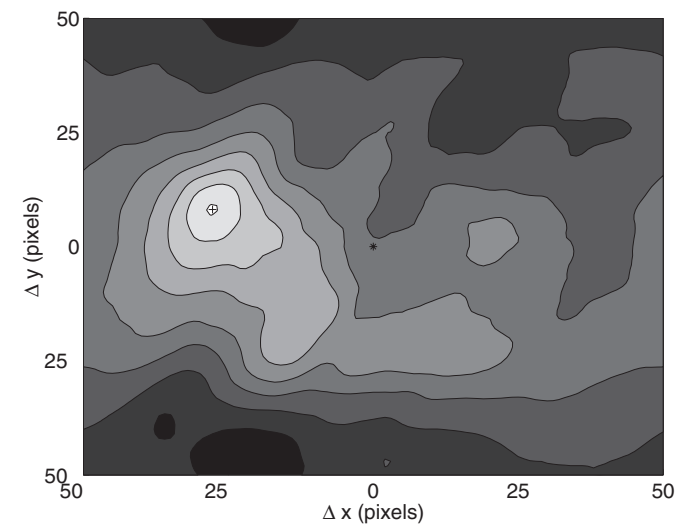

Figure 6. Shaded contour plot of the cross correlation obtained during the (first) translation phase. Note the well-defined maximum indicating the optimal translation.

The cross-correlation for the image pair shown above is presented in Figure 6. Notice that there is a well-defined peak whose position indicates the best-fit translation of the image. This peak is interpolated to sub-pixel accuracy by fitting the crosscorrelation near the maximum to a Gaussian surface (with arbitrary orientation and aspect ratio). Using this translation data, Image B is translated using a spline-based subpixel interpolation routine. In addition, a circular ring image is interpolated from the original Cartesian-based pixel grid, again using spline interpolation. A onedimensional cross-correlation in the azimuthal direction is performed on the ring image to determine the image rotation. As with the translational computation, Gaussian sub-pixel interpolation is used to optimize the rotation between the two images. The azimuthal cross-correlation is performed for several radial values, and the median rotational angle is chosen as the optimal image rotation. Image $\mathrm{B}$ is rotated using a bicubic interpolation, and the interrogation areas are re-computed. The entire process is repeated iteratively until no further improvement is observed. Additional details are reported in [5]. The results of the completed translation/rotation operation are shown in Figure 7, which shows the previous image pair, superimposed after the optimal translation and rotation. For a perfect image pair, we find that the process is accurate to better than 0.02 pixels and 0.02 degrees, although image-to-image noise does degrade this performance.

\section{CONCLUSIONS}

An IR micro-motion system designed to illuminate and measure particle and surface motions inside silicon micromachined devices using infrared wavelengths has been developed and demonstrated. The system has a resolution of approximately 360 $\mathrm{nm}$, although this can be improved by a factor of 2 with high NA optics. Although the resolution is not as good as visible-light microPIV systems (due to both the longer wavelength, the need for larger focal length optics and limits in current IR imaging technologies), it is more than adequate for many micro flows and motions of interest, particularly since other means of interrogation inside the structure are not possible. The system is now being extended to incorporate more sophisticated motion algorithms, as well as to measure instabilities, mixing and reactions inside complex silicon devices.
This work was supported by DARPA and NSF. The authors would like to acknowledge Carl Meinhart and Steve Wereley for many valuable discussions and the use of their PIV code.

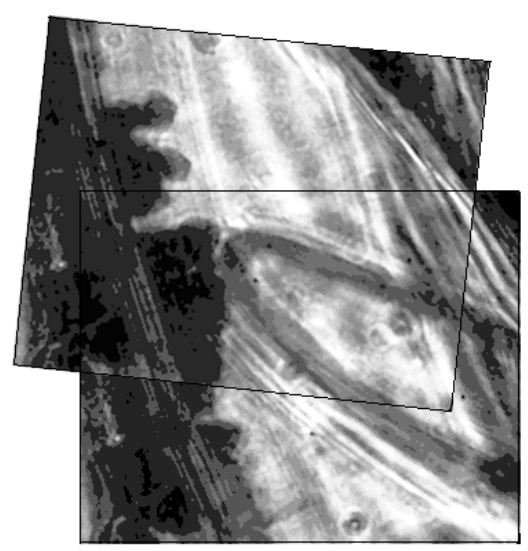

Figure 7. Microrotor image pair, translated, rotated and superimposed, indicating the optimal image shift. In this case $\Delta x$ $=-44.1, \Delta y=-111.4$ (pixels) and $\Theta=5.1$ degrees.

\section{REFERENCES}

1. RJ Adrian (1991) Particle-imaging techniques for experimental fluid mechanics. Ann. Rev. of Fluid Mech. 23: 261-301.

2. M Born \& E Wolf (1997). Principles of Optics. Sixth Edition. Cambridge University Press, Cambridge.

3. L Fréchette, SA Jacobson, KS Breuer, FE Ehrich, R Ghodssi, R Khanna, C-W Wong, X Zhang, MA Schmidt \& AHEpstein. (2000). "Demonstration of a Microfabricated High-Speed Turbine Supported on Gas Bearings" Proceedings, IEEE Solid State Sensors and Actuators Workshop. Hilton Head SC.

4. D Freeman (2001) Measuring Motions in MEMS. Materials Research Soc. Bulletin. 26: 4:305-306.

5. KS Breuer, JC Bird, G Han \& KJ Westin. (2001). "Infrared PIV for Measurement of Fluid and Solid Motion in Micromachined Devices". Proceedings, ASME IMECE New York, NY. November 2001.

6. CD Meinhart, ST Wereley \& JG Santiago (1999) PIV measurements of a microchannel flow. Exp. in fluids 27: 414419.

7. CD Meinhart, ST Wereley \& MHB Gray (2000) Volume illumination for two-dimensional particle image velocimetry. Meas. Sci. Technol. 11: 809-814.

8. JG Santiago, ST Wereley, CD Meinhart, DJ Beebe \& RJ Adrian. (1998). A particle image velocimetry system for microfluids. Exp. in Fluids 25:316-319.

9. JK Sveen (2000) MatPIV 1.4, Mechanics Division, Department of Mathematics, University of Oslo, Norway.

10. ST Wereley. (2000) Personal communication. 